\title{
Effectiveness Analysis of a Two Non-Identical Unit Standby System with Switching Device and Proviso of Rest
}

\author{
Darpandeep Kour \\ Department of Statistics, \\ University of Jammu, Jammu-180006, India \\ Corresponding author: darpandeep25@gmail.com \\ J. P. Singh Joorel \\ Department of Statistics, \\ University of Jammu, Jammu-180006, India \\ E-mail: jpsjoorel@gmail.com \\ Neha Sharma \\ Department of Statistics, \\ University of Jammu, Jammu-180006, India \\ E-mail: nehasharma131091@gmail.com
}

(Received May 11, 2019; Accepted August 31, 2019)

\begin{abstract}
This paper deals with two-unit cold standby system with switching device and proviso of rest. The system consists of two non-identical units which are connected in parallel redundancy. Initially, the first unit is in operative mode and second is kept in cold standby mode. There is also a proviso for rest for the first unit after a random time from its starting operation. For operation and repair, priority is always given to first unit. A switching device is used to shift the failed unit to repair mode. If the switching device is found non-operational then priority is given to it for repair provided that system is in functional mode. The effectiveness of the proposed system has been carried out by determining various reliability characteristics of the proposed model.
\end{abstract}

Keywords- Reliability, Availability, Effectiveness, Switching device.

\section{Introduction}

The theory of reliability studies the failure laws of systems that are operating within the predetermined limits under given operating conditions and reliability is an internal property of operating systems, and, as such, it has to be specified as part of the system characteristics. Thus, it becomes imperative to develop a quantitative basis for describing the failure process of system to measure unambiguously their reliability; this then enables one to compare the performance of similar system on a reliability basis and to develop methods for improving the reliability of a particular system. There are many methods cited in literature for achieving improved system reliability, broadly by decreasing the failure intensity of the system through technological consideration, by introducing redundancy at the component or system level, by improving the overall design of system to achieve maximum reliability within limitations (Polovko, 1968). It is evident that if in a system failed components are immediately replaced or repaired, their reliability would be maintained at all times. Therefore, effectiveness of a system can be increased by adopting good repair and maintenance policies.

A large number of authors have analyzed several models considering different types of repair and 
International Journal of Mathematical, Engineering and Management Sciences

Vol. 4, No. 6, 1496-1507, 2019

https://dx.doi.org/10.33889/IJMEMS.2019.4.6-118

maintenance policies. Based on these policies, Li et al. (1998) considered the analysis of three unit with repairable facilities and Yamashiro (1980) discussed a repairable multistage device with general repair time, whereas Chung (1987) worked on human error and common cause failures of parallel-system with standby unit. Further, Mazumdar (1970) discussed reliability analysis of two-unit redundant repairable systems with inspection after its failure and Agnihotri et al. (1993) analyzed stochastic behavior of a system with two type of failure. While, Tanino and Fukazawa (1988) presented a model of parallel unit systems with maintenance policies in Markovian process, Agarwal and Kumar (1981) analyzed a repairable system with delayed replacement, Gaver (1963) discussed the concept of mean time to system failure and availability of parallel redundant system with repair facility and Misra and Rao (1970) discussed reliability analysis of redundant networks using flow graphs. Further, Joorel et al. (1995) analyzed the behaviour of priority standby system with different types of repair facilities. However, Seo et al. (2003) has considered and discussed an algorithm to determine life time and reliability estimation of repairable redundant system. Later on, Malik and Barak (2009) worked on reliability and economic analysis of a system operating under different weather conditions. In continuity to earlier work, Chib et al. $(2014,2016)$ used the concept of proviso of rest in analyzing the system model and a two unit cold standby with partial and total failure and priority, Sharma et al. (2012) analyzed stochastic behavior of a two- unit system with partial failure and fault detection. Further, Park (2014) carried out a warranty cost analysis for multi-component systems with imperfect repair and Zheng et al. (2018) discussed the reliability importance of components with standby redundancy schemes in a real-time computing system. From the above literature, it is clear that all the models have the assumption that the repair facility is continuously available to attend to the repair of the failed unit.

Keeping in view the ongoing discussion and enhancing the reliability of the system, a two-unit standby system with switching device and proviso of rest has been proposed and analyzed in this paper. The proposed system consists of two non-identical units say $U_{1}$ and $U_{2}$ which are connected in parallel redundancy. Initially, $U_{1}$ is in operative mode and $U_{2}$ in cold standby. Each unit has only two mode- Normal and Total failure. Priority is given to $U_{1}$ over $U_{2}$ for both operation as well as repair. There is also a proviso for rest for the $U_{1}$ after a random time of its starting operation. A switching device is used to shift the failed unit to repair mode. The switching device may found in operative mode with a very high probability $p$ and in nonoperative mode with a very low probability $q$ such that $p+q=1$. If the switching device is found non-operational then priority is given to it for repair provided that system is in functional mode. To study the behaviour of the proposed model various reliability characteristics have been obtained on using regenerative point technique in Markov process.

\section{Assumptions}

The followings assumptions have been made to describe the system:

(i) The failure time and repair time of both the units are assumed to be a random variable which follows exponential distribution but with different parameters.

(ii) The repair time distribution of switching device is also taken as exponential.

(iii) The working time of both the units are assumed to be distributed arbitrary.

(iv) On completion of repair of failed unit, it becomes operational or moves to standby mode immediately as the case may be.

(v) The switching device is assumed to be perfect.

(vi) There is always a single repair facility available within the system.

(vii) After repair, a unit becomes as good as new. 
International Journal of Mathematical, Engineering and Management Sciences

Vol. 4, No. 6, 1496-1507, 2019

https://dx.doi.org/10.33889/IJMEMS.2019.4.6-118

(viii) All the random variables associated with different times are taken as independent.

\section{Notations and Possible States of the System}

The following notations have been used in describing the proposed model:

$\alpha_{1}, \alpha_{2} \quad: \quad$ Constant failure rate of the operative and cold standby unit respectively.

$\mu_{1}, \mu_{2} \quad: \quad$ Constant repair rate of the operative and cold standby unit respectively.

$f(),. F($.$) \quad : \quad$ pdf and cdf of the working time of unit-1.

$h(),. H($.$) \quad : \quad$ pdf and cdf of the working time of unit-2.

$\beta \quad$ : $\quad$ Repair rate of switching device.

$p \quad: \quad$ Probability that the switching device is perfect.

$q \quad: \quad$ Probability that the switching device is not perfect.

$U_{1 o} / U_{1 r e s t} / U_{1 r} / U_{1 R} / U_{1 w r} \quad:$ Unit-1 is in operative/rest mode/repair/repair continued/waiting for repair.

$U_{2 o} / U_{2 s} / U_{2 r} / U_{2 w r} \quad:$ Unit-2 is operative/in standby mode/is under repair/is waiting for repair.

$T_{r} \quad:$ Switching device is under repair.

The other notations and symbols are standard and have their usual meaning.

The possible states of the system are described and given in Figure 1 wherein the states $S_{1}, S_{2}, S_{4}, S_{5}$ are up states, $S_{3}$ and $S_{6}$ are down states and $S_{7}, S_{8}, S_{9}$ are failed states.

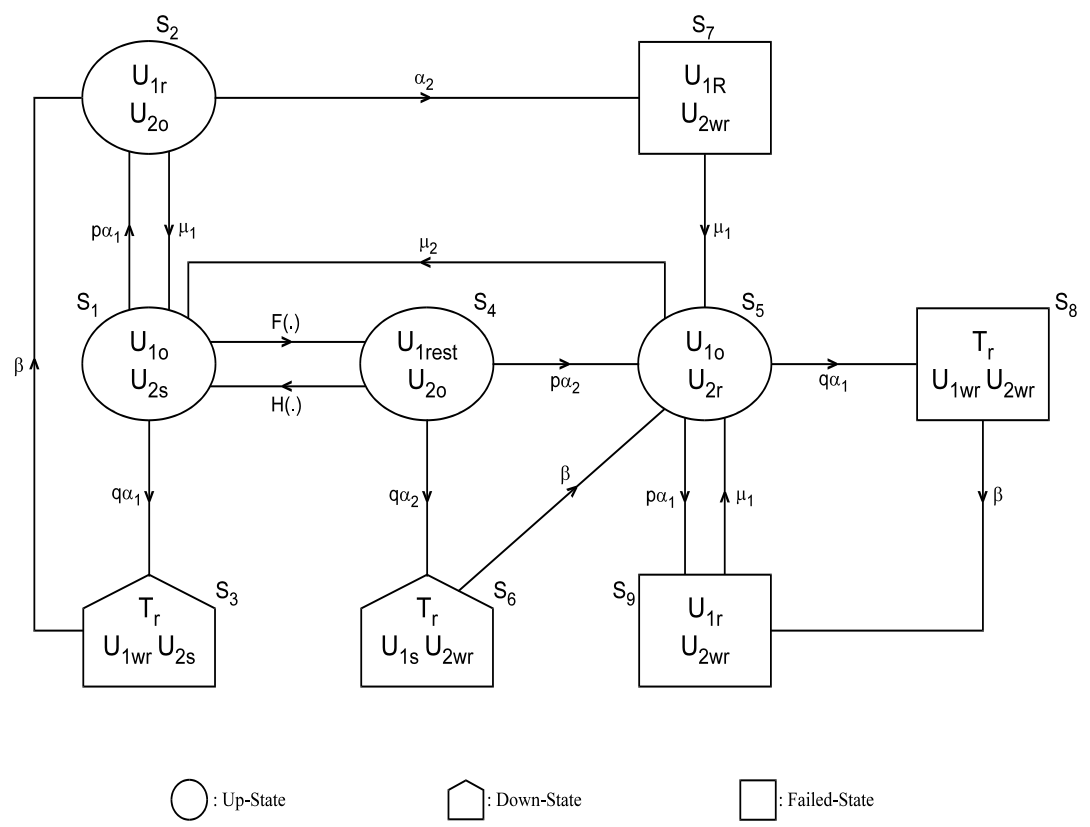

Figure 1. Transition diagram 
International Journal of Mathematical, Engineering and Management Sciences

Vol. 4, No. 6, 1496-1507, 2019

https://dx.doi.org/10.33889/IJMEMS.2019.4.6-118

\section{Transition Probabilities, Mean Sojourn Times and Mean Time to System Failure}

If $\mathrm{T}_{1}, \mathrm{~T}_{2}, \mathrm{~T}_{3} \ldots$ denote the epochs at which the system enter any state and $X_{n}$ denotes the state visited at point $T_{n+}$, i.e. just after the transition at $T_{n}$ then the transient and steady state transition probabilities are defined as $\mathrm{Q}_{\mathrm{ij}}(\mathrm{t})=\mathrm{P}\left[\mathrm{X}_{\mathrm{n}+1}=\mathrm{j}, \mathrm{T}_{\mathrm{n}+1}-\mathrm{T}_{\mathrm{n}} \leq \mathrm{t} \mid \mathrm{X}_{\mathrm{n}}=\mathrm{i}\right]$ and $p_{i j}=\lim _{t \rightarrow \infty} Q_{i j}(t)$ respectively. The following steady state transition probabilities of the system are obtained:

$$
\begin{aligned}
& p_{12}=p\left(1-\tilde{F}\left(\alpha_{1}\right)\right) \\
& p_{13}=q\left(1-\tilde{F}\left(\alpha_{1}\right)\right) \\
& p_{14}=\tilde{F}\left(\alpha_{1}\right) \\
& p_{21}=\frac{\mu_{1}}{\mu_{1}+\alpha_{2}} \\
& p_{27}=\frac{\alpha_{2}}{\mu_{1}+\alpha_{2}} \\
& p_{32}=p_{65}=p_{75}=p_{89}=p_{95}=1 \\
& p_{41}=\widetilde{H}\left(\alpha_{2}\right) \\
& p_{45}=p\left[1-\widetilde{H}\left(\alpha_{2}\right)\right] \\
& p_{46}=q\left[1-\widetilde{H}\left(\alpha_{2}\right)\right] \\
& p_{51}=\frac{\mu_{2}}{\mu_{2}+\alpha_{1}} \\
& p_{58}=q \frac{\alpha_{1}}{\mu_{2}+\alpha_{1}} \\
& p_{59}=p \frac{\alpha_{1}}{\mu_{2}+\alpha_{1}}
\end{aligned}
$$

It may be noted that $\sum_{j} p_{i j}=1$, for all possible values of $i$.

Further, if $T_{i}$ denotes the sojourn time in state $S_{i}$ then mean sojourn time in $S_{i}$ is denoted by $\psi_{i}$ which is defined as $\psi_{i}=E[T]=\int P\left(T_{i}>t\right) d t$, then the following mean sojourn times in different states are obtained:

$$
\begin{aligned}
& \psi_{1}=\frac{1-\tilde{F}\left(\alpha_{1}\right)}{\alpha_{1}} \\
& \psi_{2}=\frac{1}{\mu_{1}+\alpha_{2}} \\
& \psi_{3}=\frac{1}{\beta} \\
& \psi_{4}=\frac{1-\widetilde{H}\left(\alpha_{2}\right)}{\alpha_{2}} \\
& \psi_{5}=\frac{1}{\mu_{2}+\alpha_{1}} \\
& \psi_{6}=\psi_{8}=\frac{1}{\beta}
\end{aligned}
$$


International Journal of Mathematical, Engineering and Management Sciences

Vol. 4, No. 6, 1496-1507, 2019

https://dx.doi.org/10.33889/IJMEMS.2019.4.6-118

$\psi_{7}=\psi_{9}=\frac{1}{\mu_{1}}$

To obtained the mean time to system failure let $T_{i}$ denotes as the time to system failure and $\pi_{i}(t)$ denotes as the cdf of time to system failure for the first time when the system start operation from state $S_{i}$. To obtain the distribution function of time to system failure, we consider the failed states as absorbing state. The following expression of the mean time to system failure is obtained which may easily be verified:

$$
M T S F=\frac{\left(\mu_{1}+\alpha_{2}\right)\left(\mu_{2}+\alpha_{1}\right)\left[\alpha_{2}-\alpha_{2} \tilde{F}\left(\alpha_{1}\right)+\alpha_{1} \tilde{F}\left(\alpha_{1}\right)\left(1-\widetilde{H}\left(\alpha_{2}\right)\right]+\alpha_{1} \alpha_{2} p\left(\mu_{2}+\alpha_{1}\right)\right.}{\left[1-\tilde{F}\left(\alpha_{1}\right)\right]+\alpha_{1} \alpha_{2} p\left(\mu_{1}+\alpha_{2}\right)\left[1-\widetilde{H}\left(\alpha_{2}\right)\right]}
$$

\section{Availability Analysis}

As we know that the availability of the system which is denoted by $A_{i}(t)$, is defined as the probability that the system is initially in upstate $S_{i}$ and available at time $t$. Therefore, by simple probabilistic arguments, the steady state availability of the system is obtained and given by

$$
\begin{gathered}
\mu_{1} \beta\left\{[ 1 - \tilde { F } ( \alpha _ { 1 } ) ] \left[\alpha_{2}\left(\mu_{1}+\alpha_{2}\right)\left(\mu_{2}+q \alpha_{1}\right)+\alpha_{1} \alpha_{2} \mu_{2}+\alpha_{1} \alpha_{2}^{2}\left(\mu_{1}+\alpha_{2}\right)+\tilde{F}\left(\alpha_{1}\right)\left[1-\widetilde{H}\left(\alpha_{2}\right)\right]\right.\right. \\
A_{1}=\frac{\left.\left[\alpha_{1} \mu_{2}\left(\mu_{1}+\alpha_{2}\right)+\alpha_{1} \alpha_{2}\left(\mu_{1}+\alpha_{2}\right)\left(\mu_{2}+\alpha_{1}\right)\right]\right\}}{\left[1-\tilde{F}\left(\alpha_{1}\right)\right]\left[\alpha_{2} \mu_{1} \mu_{2} \beta\left(\mu_{1}+\alpha_{2}\right)+2 \alpha_{1} \alpha_{2} \mu_{2}+q \alpha_{1} \alpha_{2} \mu_{1} \mu_{2}-\alpha_{1}^{2} \alpha_{2} \mu_{1} \beta\right]+\widetilde{F}\left(\alpha_{1}\right)\left[\alpha_{1} \mu_{1} \mu_{2} \beta\left(1-\widetilde{H}\left(\alpha_{2}\right)\right)\right.} \\
+\alpha_{1} \alpha_{2} \mu_{1}^{2} \beta-\alpha_{1} \alpha_{2} \mu_{1} \beta \widetilde{H}\left(\alpha_{2}\right)\left(\mu_{1}+\alpha_{2}\right)+\alpha_{1} \alpha_{2} \mu_{1} \mu_{2} q\left(1-\widetilde{H}\left(\alpha_{2}\right)\right)\left(\mu_{1}+\alpha_{2}\right)+\alpha_{1}^{2} \mu_{1}^{2} q-\alpha_{1}^{2} \alpha_{2} \mu_{1} q \widetilde{H}\left(\alpha_{2}\right) \\
\left.\left(\mu_{1}+\alpha_{2}\right)-\alpha_{1}^{2} \alpha_{2} \beta \widetilde{H}\left(\alpha_{2}\right)\left(\mu_{1}+\alpha_{2}\right)\right]+\alpha_{1} \alpha_{2}\left[\alpha_{2} \mu_{1} \beta+q \alpha_{1} \alpha_{2} \mu_{1}+\alpha_{1} \beta\left(\mu_{1}+\alpha_{2}\right)\right]
\end{gathered}
$$

\section{Busy Period Analysis}

Consider, $B_{i}(t)$ is defined as the probability that the system started from regenerative state $S_{i} \epsilon E$ at time $t=0$, is under repair at time $t$ because of the failure of the unit. Therefore, in steady state this probability is given by

$$
\begin{gathered}
\alpha_{1} \alpha_{2}\left\{\left[1-\tilde{F}\left(\alpha_{1}\right)\right]\left[\mu_{1} \mu_{2} \beta+\mu_{1} \mu_{2} q\left(\mu_{1}+\alpha_{2}\right)+\mu_{1} \alpha_{2} \beta+\mu_{2} \alpha_{2} \beta\right]+\widetilde{F}\left(\alpha_{1}\right)\left[\left(\mu_{1}+\alpha_{2}\right)\right.\right. \\
\left.\left(\mu_{1} \beta+\mu_{1} \mu_{2} q\right)-\mu_{1} \beta\left(\mu_{2}+\alpha_{1}\right)\left(1-\mu_{1}\right)+\alpha_{1} \mu_{1}^{2} q\right]-\tilde{F}\left(\alpha_{1}\right) \widetilde{H}\left(\alpha_{2}\right)\left[( \mu _ { 1 } + \alpha _ { 2 } ) \left(\mu_{1} \beta+\right.\right. \\
B_{1}=\frac{\left.\left.\left.\mu_{1} \mu_{2} q-\beta\right)+\left(\mu_{1}+\alpha_{2}\right)\left(\mu_{2}+\alpha_{1}\right)\left(\mu_{1} \alpha_{1} q-\beta\right)\right]+\alpha_{1} \alpha_{2}\left(\mu_{1} q+\beta\right)\right\}}{\left[1-\tilde{F}\left(\alpha_{1}\right)\right]\left[\alpha_{2} \mu_{1} \mu_{2} \beta\left(\mu_{1}+\alpha_{2}\right)+2 \alpha_{1} \alpha_{2} \mu_{2}+q \alpha_{1} \alpha_{2} \mu_{1} \mu_{2}-\alpha_{1}^{2} \alpha_{2} \mu_{1} \beta\right]+\tilde{F}\left(\alpha_{1}\right)\left[\alpha_{1} \mu_{1} \mu_{2} \beta\left(1-\widetilde{H}\left(\alpha_{2}\right)\right)\right.} \\
+\alpha_{1} \alpha_{2} \mu_{1}^{2} \beta-\alpha_{1} \alpha_{2} \mu_{1} \beta \widetilde{H}\left(\alpha_{2}\right)\left(\mu_{1}+\alpha_{2}\right)+\alpha_{1} \alpha_{2} \mu_{1} \mu_{2} q\left(1-\widetilde{H}\left(\alpha_{2}\right)\right)\left(\mu_{1}+\alpha_{2}\right)+\alpha_{1}^{2} \mu_{1}^{2} q-\alpha_{1}^{2} \alpha_{2} \mu_{1} q \widetilde{H}\left(\alpha_{2}\right) \\
\left.\left(\mu_{1}+\alpha_{2}\right)-\alpha_{1}^{2} \alpha_{2} \beta \widetilde{H}\left(\alpha_{2}\right)\left(\mu_{1}+\alpha_{2}\right)\right]+\alpha_{1} \alpha_{2}\left[\alpha_{2} \mu_{1} \beta+q \alpha_{1} \alpha_{2} \mu_{1}+\alpha_{1} \beta\left(\mu_{1}+\alpha_{2}\right)\right]
\end{gathered}
$$

\section{Expected Number of Visits by the Repair Facilities}

Consider $V_{i}(t)$ as the expected number of visits by the repairman during the time interval $(0, t]$ when the system initially starts from regenerative state $S_{i}$, thus in the long run the expected number of visits when the system starts its operation from state 1 are given as

$$
\begin{aligned}
& V_{1}= \frac{\alpha_{1} \alpha_{2} \mu_{1} \mu_{2}^{2} \beta\left(\mu_{1}+\alpha_{2}\right)\left[1-\widetilde{F}\left(\alpha_{1}\right) \widetilde{H}\left(\alpha_{2}\right)\right]}{\left[1-\tilde{F}\left(\alpha_{1}\right)\right]\left[\alpha_{2} \mu_{1} \mu_{2} \beta\left(\mu_{1}+\alpha_{2}\right)+2 \alpha_{1} \alpha_{2} \mu_{2}+q \alpha_{1} \alpha_{2} \mu_{1} \mu_{2}-\alpha_{1}^{2} \alpha_{2} \mu_{1} \beta\right]+\tilde{F}\left(\alpha_{1}\right)\left[\alpha_{1} \mu_{1} \mu_{2} \beta\left(1-\widetilde{H}\left(\alpha_{2}\right)\right)\right.} \\
&+\alpha_{1} \alpha_{2} \mu_{1}^{2} \beta-\alpha_{1} \alpha_{2} \mu_{1} \beta \widetilde{H}\left(\alpha_{2}\right)\left(\mu_{1}+\alpha_{2}\right)+\alpha_{1} \alpha_{2} \mu_{1} \mu_{2} q\left(1-\widetilde{H}\left(\alpha_{2}\right)\right)\left(\mu_{1}+\alpha_{2}\right)+\alpha_{1}^{2} \mu_{1}^{2} q-\alpha_{1}^{2} \alpha_{2} \mu_{1} q \widetilde{H}\left(\alpha_{2}\right) \\
&\left.\left(\mu_{1}+\alpha_{2}\right)-\alpha_{1}^{2} \alpha_{2} \beta \widetilde{H}\left(\alpha_{2}\right)\left(\mu_{1}+\alpha_{2}\right)\right]+\alpha_{1} \alpha_{2}\left[\alpha_{2} \mu_{1} \beta+q \alpha_{1} \alpha_{2} \mu_{1}+\alpha_{1} \beta\left(\mu_{1}+\alpha_{2}\right)\right]
\end{aligned}
$$


International Journal of Mathematical, Engineering and Management Sciences

Vol. 4, No. 6, 1496-1507, 2019

https://dx.doi.org/10.33889/IJMEMS.2019.4.6-118

\section{Profit Function Analysis}

The cost analysis of the system can also be studied through a cost function of the system by considering expected uptime of the system, expected busy period of the repair facility and expected number of visits by the repair facility.

Let the expected profit incurred by the system during time $(0, t]$ is denoted by $P(t)$ which is defined as

$P(t)=$ Expected total revenue in time $(0, t]$ - total expected service cost in time $(0, t]-$ expected cost of vists of the repair facility in time $(0, t]$

$$
=R \mu_{u p}(t)-C_{1} \mu_{b}(t)-C_{2} V_{1}(t)
$$

where,

$\mu_{u p}(t)=$ expected uptime of the system in time $(0, t]$ which is given by $\int_{0}^{t} A_{1}(u) d u$ such that,

$$
\mu_{u p}^{*}(s)=A_{1}^{*}(s) / s
$$

$\mu_{b}(t)=$ expected duration of busy time of the repair facility in time $(0, t]$ and is given by $\int_{0}^{t} B_{1}(u) d u$ such that, $\mu_{b}^{*}(s)=B_{1}^{*}(s) / s$

$\mathrm{R}=$ revenue generated by the system in time $(0, t]$

$C_{1}=$ cost incurred in time $(0, t]$ when repair facility remains busy

$C_{2} \quad=$ per visit cost of the repair facility

Therefore, the expected profit per unit of time in steady state becomes

$P=\lim _{t \rightarrow \infty} P(t)=R A_{1}-C_{1} B_{1}-C_{2} V_{1}$

where, $A_{1}, B_{1}$ and $V_{1}$ are the availability, busy period and expected number of visits in steady state which are given by equations (21), (22) and (23) respectively.

\section{Graphical Study of the System Model}

To study the graphical behaviour of the system, we plot the MTSF, Availability and Profit function with respect to the failure rate of both the units for different values of repair rate.

In Figure 2, the MTSF w.r.t. $\alpha_{1}$ is plotted by taking different values of $\mu_{1}$ as $0.6,1.2$ and 1.8 and fixing the other parameters as $\alpha_{2}=0.69, \mu_{2}=0.8, \gamma_{1}=0.5, \gamma_{2}=0.25, \beta=0.45$. It is observed that the MTSF decreases with increase in the failure rate $\alpha_{1}$ for different values of $\mu_{1}$ and increase with increase in repair rate $\mu_{1}$. Similarly, In Figure 3, the graph of MTSF is plotted w.r.t $\alpha_{1}$ for different values of $\mu_{2}$ as $0.6,1.2$ and 1.8 and fixed the other parameters. In Figure 4, we plot the MTSF w.r.t. $\alpha_{2}$ for different values of $\mu_{1}$ as $0.8,1.6$ and 2.4 and fixed the other parameters $\alpha_{1}=0.69, \mu_{2}=0.8, \gamma_{1}=0.5, \gamma_{2}=0.25, \beta=0.45$. Similarly, In Figure 5, we plot the MTSF w.r.t. $\alpha_{1}$ for different values of $\mu_{2}$ as $0.8,1.6$ and 2.4 and fixed the other parameters.

The availability w.r.t. $\alpha_{1}$ for various values of $\mu_{1}$ as $0.4,0.8$ and 1.2 and fixed the other parameters $\alpha_{2}=0.65, \mu_{2}=0.9, \gamma_{1}=0.6, \gamma_{2}=0.26, \beta=0.48$ has been graphed in Figure 6, wherein it is observed that the availability decreases with increase in the failure rate $\alpha_{1}$ for different values of $\mu_{1}$ and increase with increase in repair rate $\mu_{1}$. Similarly, In Figure 7, we plot the availability w.r.t. $\alpha_{1}$ for different values of $\mu_{2}$ as $0.4,0.8$ and 1.2 and fixed the other parameters. In Figure 8, we plot the availability w.r.t. $\alpha_{2}$ for different values of $\mu_{1}$ as $0.7,1.4$ and 
International Journal of Mathematical, Engineering and Management Sciences

Vol. 4, No. 6, 1496-1507, 2019

https://dx.doi.org/10.33889/IJMEMS.2019.4.6-118

2.1 and fixed the other parameters $\alpha_{1}=0.72, \mu_{2}=0.32, \gamma_{1}=0.8, \gamma_{2}=0.30, \beta=0.49$. Similarly, In Figure 9, the availability w.r.t. $\alpha_{1}$ for different values of $\mu_{2}$ as $0.7,1.4$ and 2.1 by fixing the other parameters have been plotted.

And finally, the profit w.r.t. $\alpha_{1}$ for different values of $\mu_{1}$ as $0.5,1.0$ and 1.5 and fixed the other parameters $\alpha_{2}=0.70, \mu_{2}=0.8 \gamma_{1}=0.6, \gamma_{2}=0.27, \beta=0.44$ has been graphed in Figure 10. It is observed that the profit decreases with increase in the failure rate $\alpha_{1}$ for different values of $\mu_{1}$ and increase with increase in repair rate $\mu_{1}$. Similarly, In Figure 11, we plot the profit w.r.t. $\alpha_{2}$ for different values of $\mu_{2}$ as $0.5,1.0$ and 1.5 and fixed the other parameters.

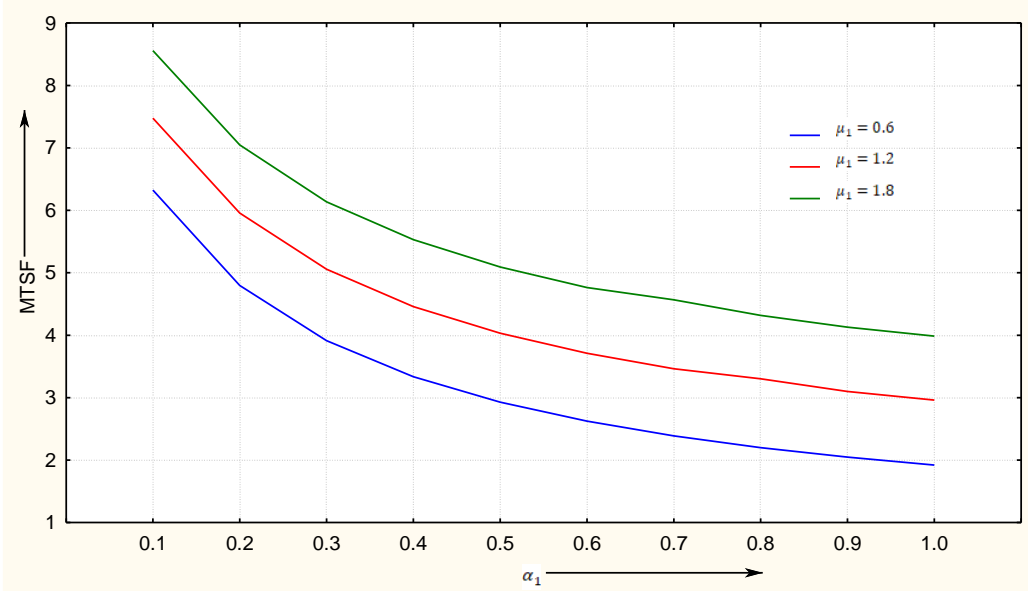

Figure 2. Behaviour of MTSF w.r.t $\alpha_{1}$ for different values of $\mu_{1}$

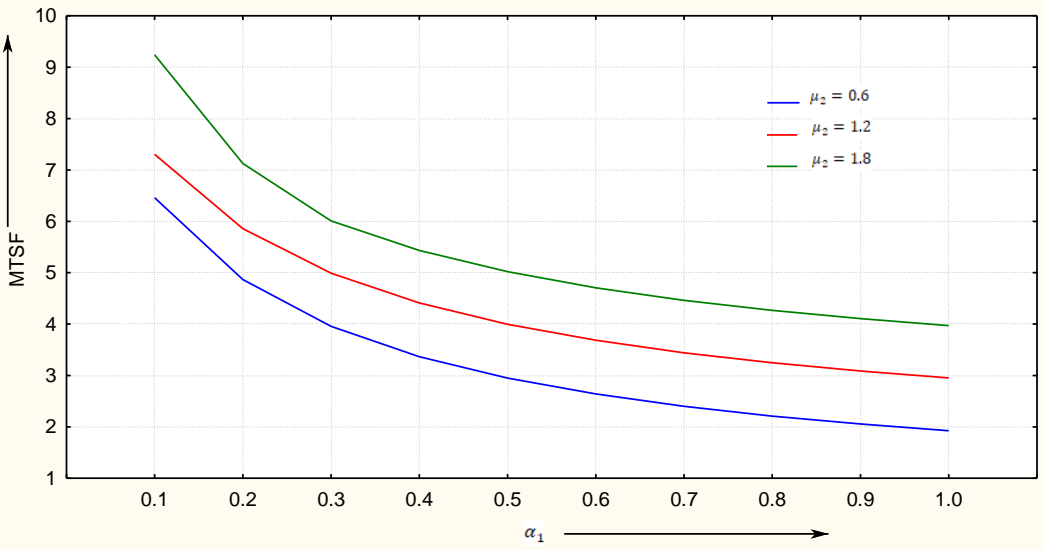

Figure 3. Behaviour of MTSF w.r.t $\alpha_{1}$ for different values of $\mu_{2}$ 
International Journal of Mathematical, Engineering and Management Sciences

Vol. 4, No. 6, 1496-1507, 2019

https://dx.doi.org/10.33889/IJMEMS.2019.4.6-118

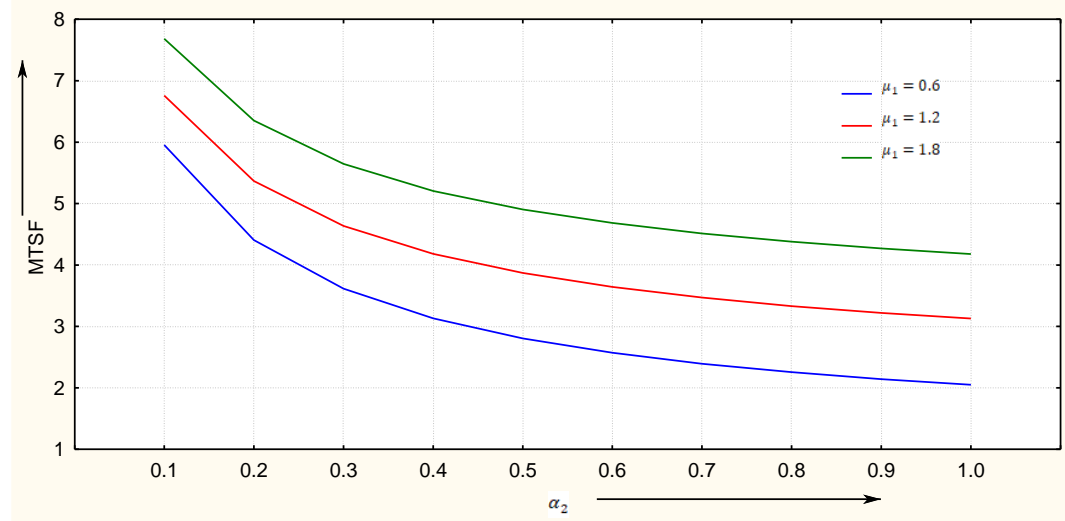

Figure 4. Behaviour of MTSF w.r.t $\alpha_{2}$ for different values of $\mu_{1}$

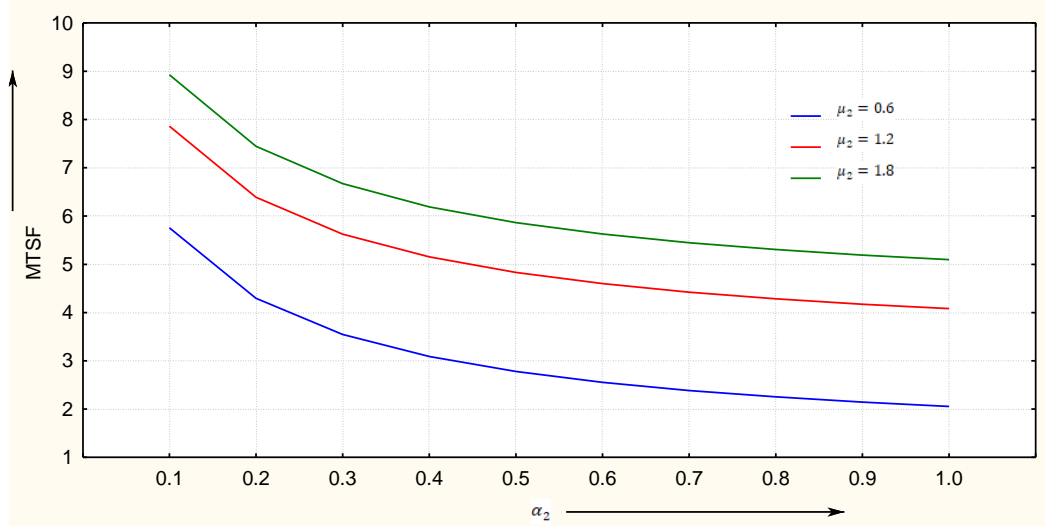

Figure 5. Behaviour of MTSF w.r.t $\alpha_{2}$ for different values of $\mu_{2}$

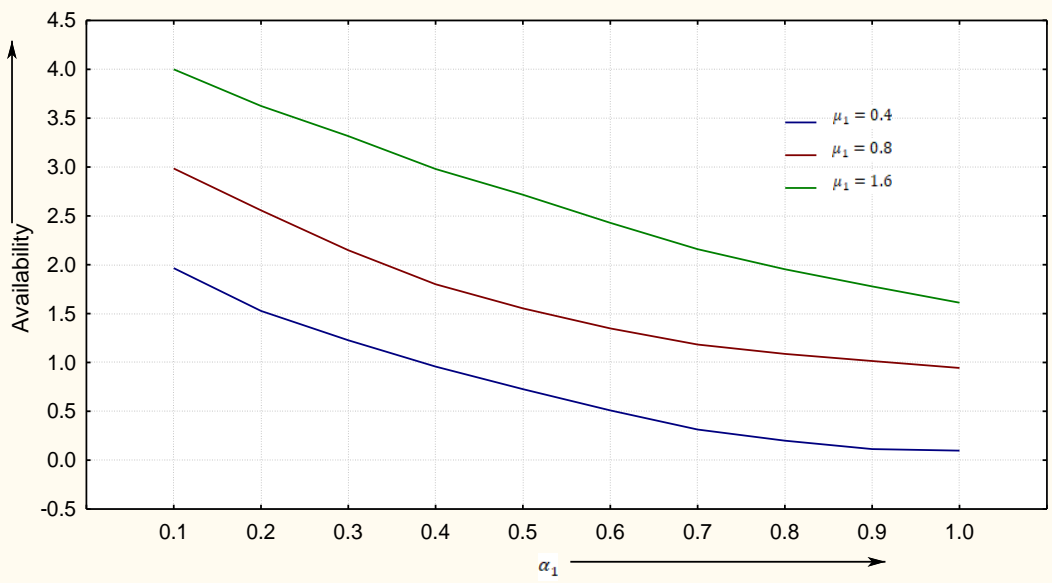

Figure 6. Behaviour of availability w.r.t $\alpha_{1}$ for different values of $\mu_{1}$ 
International Journal of Mathematical, Engineering and Management Sciences

Vol. 4, No. 6, 1496-1507, 2019

https://dx.doi.org/10.33889/IJMEMS.2019.4.6-118

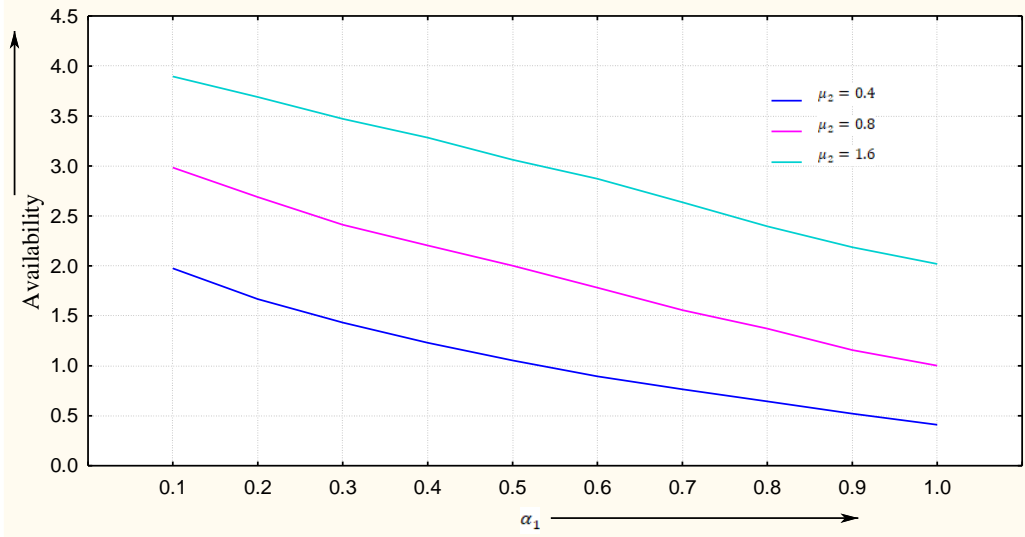

Figure 7. Behaviour of availability w.r.t. $\alpha_{1}$ for different values of $\mu_{2}$

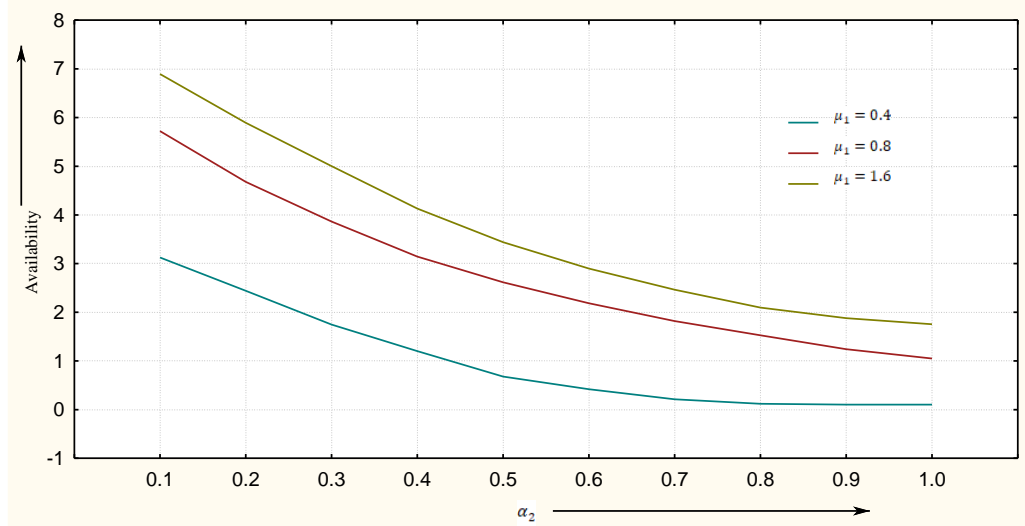

Figure 8. Behaviour of availability w.r.t $\alpha_{2}$ for different values of $\mu_{1}$

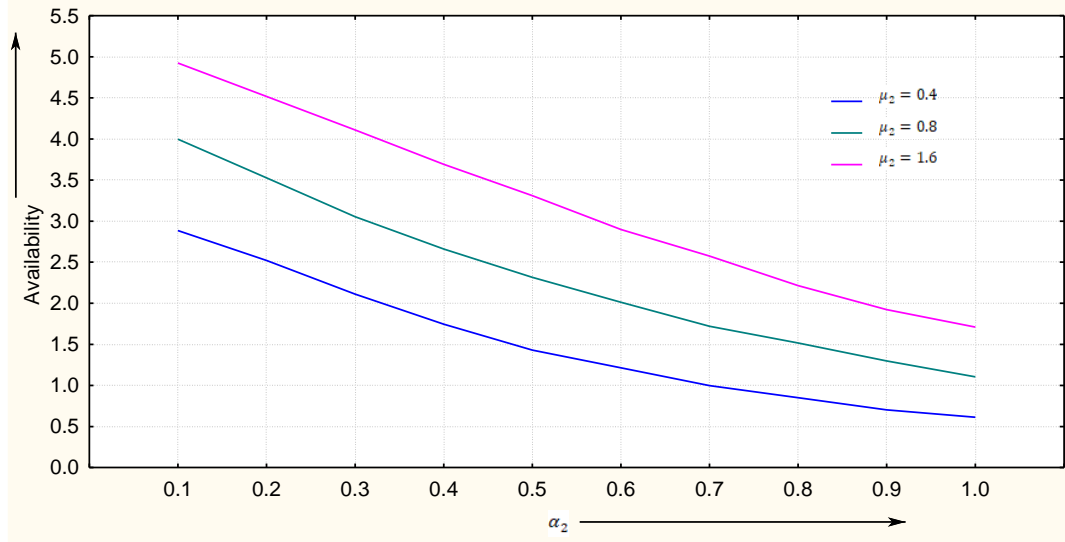

Figure 9. Behaviour of availability w.r.t $\alpha_{2}$ for different values of $\mu_{2}$ 
International Journal of Mathematical, Engineering and Management Sciences

Vol. 4, No. 6, 1496-1507, 2019

https://dx.doi.org/10.33889/IJMEMS.2019.4.6-118

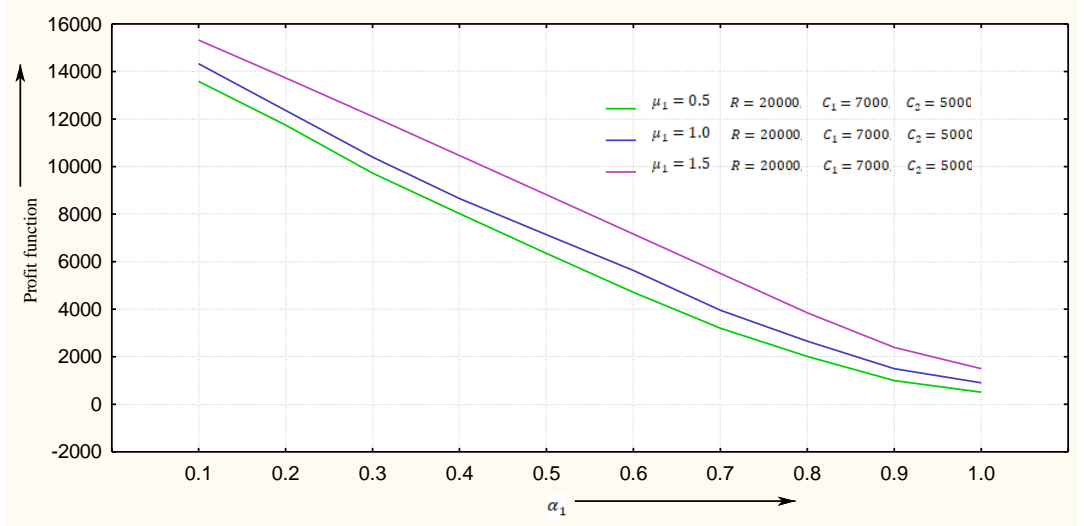

Figure 10. Behaviour of profit function w.r.t $\alpha_{1}$ for different values of $\mu_{1}$

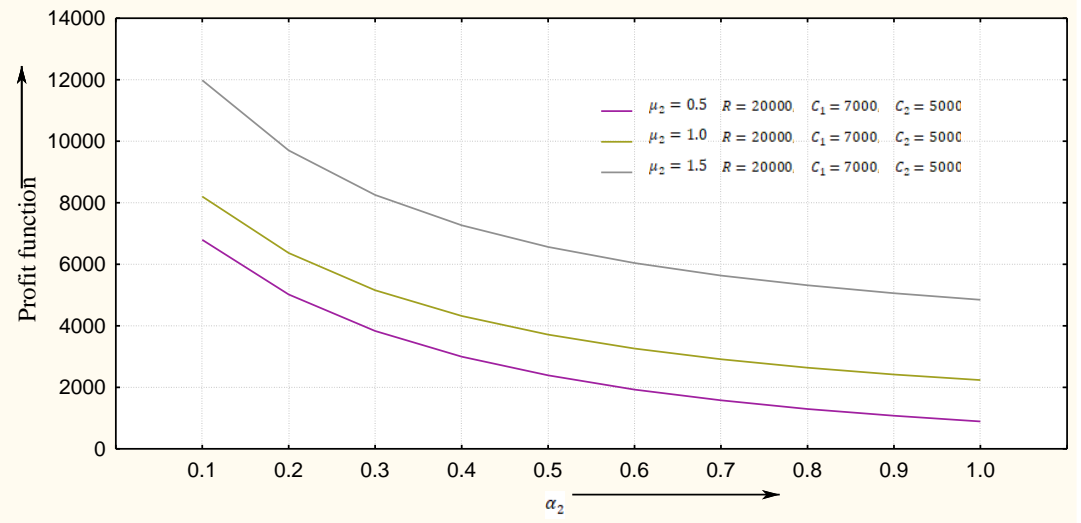

Figure 11. Behaviour of profit function w.r.t $\alpha_{2}$ for different values of $\mu_{2}$

\section{Conclusion}

It is observed that the mean time to system failure, availability along with the profit of the system decreases uniformly as fixing the other parameters, the failure rate of the system increases rapidly. However, it is also observed that with increase in repair rates, MTSF, availability and the profit function increases. Therefore, we conclude that by decreasing failure rate and increasing repair rate of the units, the expected life of the system can be increased which thus improve the reliability and the effectiveness of the system.

\section{Conflict of Interest}

It is clarified that there is no conflict of interest for this publication. 
International Journal of Mathematical, Engineering and Management Sciences

Vol. 4, No. 6, 1496-1507, 2019

https://dx.doi.org/10.33889/IJMEMS.2019.4.6-118

\section{Acknowledgement}

The authors acknowledge their appreciation to the reviewers of the paper for their comments and suggestions. The first and third authors are thankful to the University of Jammu, Jammu for supporting their research work.

\section{References}

Agarwal, M., \& Kumar, A. (1981). Analysis of a repairable redundant system with delayed replacement. Microelectronics Reliability, 21(2), 165 - 171.

Agnihotri, R.K., Kumar, V., \& Asari, M.A. (1993). Stochastic analysis of a redundant system with two type of failure. Microelectronics Reliability, 33(10), 1467-1471.

Chib, R., Joorel, J.P.S., \& Sharma, V. (2014). Profit analysis of a two-unit priority system with two types of repair facility and proviso of rest. International Journal of Statistics and Reliability Engineering, 1(1), 69-81.

Chib, R., Joorel, J.P.S., \& Sharma, V. (2016): Analysis of a two non-identical unit cold standby system with partial and total failure and priority, Proceedings of the 10th INDIACom-2016; IEEE Conference ID: 37465 International Conference on Computing for Sustainable Global Development, 6304-6308.

Chung, W.K. (1987). Reliability analysis of a repairable parallel-system with standby involving human error and common cause failures. Microelectronics Reliability, 27(2), 269-271.

Gaver, D.P. (1963). Time to failure and availability of a parallel system with repair. IEEE Transactions on Reliability, 12(2), 30-38.

Joorel, J.P.S., Parmar, Y.S., \& Heydari, P.D. (1995). Behaviour of priority standby redundant system with different types of repair facilities. Microelectronics Reliability, 35(5), 833-836.

Li, W., Alfa, A.S., \& Zhao, Y.Q. (1998). Stochastic analysis of a repairable system with three units and two repair facilities. Microelectronics Reliability, 38(4), 585-595.

Malik, S.C., \& Barak, M.S. (2009). Reliability and economic analysis of a system operating under different weather conditions. In: Proceedings of the National Academy of Sciences India Section A - Physical Sciences, 79(2), 205-213.

Mazumdar, M. (1970). Reliability of two-unit redundant repairable systems when failures are revealed by inspections. Society for Industrial and Applied Mathematics Journal on Applied Mathematics, 19(4), $637-647$.

Misra K.B., \& Rao, T.S.M. (1970). Reliability analysis of redundant networks using flow graphs. IEEE Transaction on Reliability, 19(1), 19-24.

Park, M. (2014). Warranty cost analysis for multi-component systems with imperfect repair. International Journal of Reliability and Applications 15(1), 51-64.

Polovko, A.M. (1968). Fundamentals of reliability theory. Academic Press, Inc, New York.

Seo, J.H., Jang, J.S., \& Bai, D.S. (2003). Life time and reliability estimation of repairable redundant system subject to periodic alternation. Reliability Engineering \& System Safety, 80(2), 197-204.

Sharma, V., Joorel, J.P.S., Chib, R., \& Bharti, R. (2012). Two-unit cold standby priority system with fault direction and provision of rest. International Journal of Research in Computer Application and Management, 2(6), 61-67. 
International Journal of Mathematical, Engineering and Management Sciences

Vol. 4, No. 6, 1496-1507, 2019

https://dx.doi.org/10.33889/IJMEMS.2019.4.6-118

Tanino, T., \& Fukazawa, F. (1988). Maintenance policies in Markovian deterioration models of parallel unit systems. International Journal of Systems Science, 19(8), 1655-1662.

Yamashiro, M. (1980). A repairable multistage device with general repair time. IEEE Transactions on Reliability, 29(3), 276.

Zheng, J., Okamura, H., \& Dohi, T. (2018). Reliability importance of components in a real-time computing system with standby redundancy schemes. International Journal of Mathematical, Engineering and Management Sciences, 3(2), 64-89. 